\title{
A Multi-exposure Images Fusion Approach for Very Large Dynamic Range Scenes
}

\author{
Chunmeng Wang and Changhe Tu \\ School of Computer Science and Technology, Shandong University \\ Jinan, Shandong, China, 250100 \\ wchm87@qq.com,chtu@sdu.edu.cn
}

\begin{abstract}
The exposure fusion technique is very useful for directly fusing multi-exposure images into a high quality image, without the physically based high dynamic range (HDR) production step. In this paper, we present an improved method for multi-exposure images fusion using multi-resolution Laplacian pyramid weighted blending. We propose a Weight Modification Factor (WMF) to modify the original weight map for each image guided by contrast, saturation and well-exposedness of each pixel in the image, and to enhance the weight of the informative pixels in ultra-bright or ultra-dark areas. Compared with previous methods, our approach preserves more useful details for scenes with very large dynamic range and achieves no artifacts. Several objective quality metrics prove the advantages of our method.
\end{abstract}

Keywords: high dynamic range image, HDR, exposure fusion, Laplacian pyramid

\section{Introduction}

Dynamic range of a real scene is extremely large, up to $10^{8}: 1$ from the bright sun to the dark shadow. The dynamic range of human eyes perception is about $10^{4}: 1$. However, the capacity of dynamic range of a low cost image capture or display device is very limited, typically only $10^{2}: 1$. Thus, a single digital image cannot show the entire dynamic range of a real scene. Fortunately, under the development of HDR technologies [1, 2], high realistic images with a wide range of luminance information could be presented on low cost devices.

Although some new specialized HDR capture devices have been developed [3-5], so far they are still far from popularity because of the high prices and hardware requirements. In order to display more colors and detail information of the over-exposed and under-exposed areas on common home-use devices, the common approach is to capture sequential low dynamic range (LDR) images with a standard digital camera by setting different exposure levels, then estimate the camera response curve and recover a HDR radiance map [6], finally display the HDR radiance map on common LDR monitors by tone mapping algorithms [7] $[8-10,16]$.

However, these typical HDR imaging methods need the camera response curve calibration. Moreover, the generated HDR image could not be directly displayed on common devices unless a tone mapping algorithm running on it, this will increase the cost of computation time and memory requirement of the device when displaying an image. Due to these reasons, exposure fusion was proposed later. It directly fuses multi-exposure images into a single high-quality composite image which could be displayed on LDR monitors, with no intermediate HDR image acquisition and no tone mapping algorithms either.

In this paper we propose an improved approach based on the multi-resolution exposure fusion method Mertens, et al., [12] to avoid the disadvantage of the method. We obtain 
weight maps guided by quality measures and blend for each Laplacian pyramid level separately to eliminate seams introduced when using the direct weighted average. Unlike previous work, we propose a Weight Modification Factor (WMF) to modify the weight of Laplacian pyramid coefficients, making the weights of informative pixels dominant even if on the high level pyramid. We use the new weight computation method to extract more visual information without halos in ultra-bright and ultra-dark areas than previous methods. (Figure 1)

The organization of the paper is as follows. In Section 2 we summarize the related work about exposure fusion. In Section 3 we analyze the characteristic of the method in [12], and explain the reason why the method does not work in ultra-bright or ultra-dark regions. We explain our method in Section 4 and show some experimental results and comparisons with previous methods in Section 5. In Section 6, we make a conclusion for our method and propose our future work.

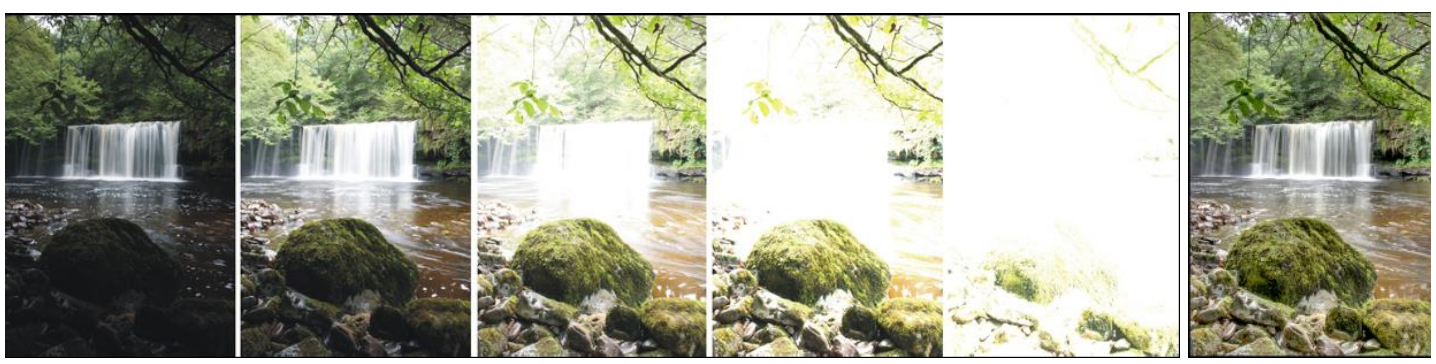

Figure 1. Five Input Pictures of Different Exposures and the Fused Image by our Method (Right)

\section{Related Work}

Many exposure fusion methods have been proposed recently [11-15]. They simplify the conventional HDR imaging pipeline and directly fuse bracketed exposures into a high-quality LDR image. Goshtasby [11] proposed a fusion method that partitions the image domain into uniform blocks, then for each block selects the image that contains the most information within that block by computing the entropy of each input color image, and then fuses the selected images to a new image with Gaussian function. Unfortunately, the method may lead to artifacts near boundaries of regions with different brightness because one block may cross different regions. Raman and Chaudhuri [13] proposed an exposure fusion method with the technique of edgepreserving bilateral filtering. The method is computationally efficient but with unsatisfactory results on color visibility. Zhao Ying, et al., [14] proposed a detailpreserving multi-exposure image fusion approach using the subband architecture. The method preserves much more details than previous methods but the gain control maps increase the excess details by enhancing the contrast. Wei Zhang, et al., [15] composed multiple images with different exposures in both static and dynamic scenes with the gradient-based quality measures considering that the gradient magnitude implies exposure quality and decreases gradually as the image approaching over-exposure or under-exposure. But the result looks unnatural because light and shade effects of the original scene are ignored. The most attractive method was proposed by Mertens, et al., [12], which determines the weight map of each image according to contrast, saturation and well-exposedness. There are obvious seams by naive weighted average blending due to sharp variations in the weight map. Blurring the weight map using a Gaussian kernel or bilateral filtering removes the seams, but introduces halos around edges. 
Therefore, on the basis of weight maps, they adopt multi-resolution Laplacian pyramid [17] weighted blending to eliminate seams and halos and achieved perfect results in most cases. However, when the dynamic range of the real scene is extremely large and contains ultra-bright and ultra-dark areas where only a small amount of details can be captured in spite of adjusting the camera exposure parameter, the method will lose the small amount of useful details in the final fused image. The reason for this problem is that the weights of informative pixels are not dominated anymore because of the weight map blurring. We will elaborate more details about this in Section 3.

\section{Analysis of Multi Resolution Exposure Fusion}

Based on the multi resolution theory, Mertens, et al., [12] proposed a method to decompose each input image into Laplacian pyramid, build the normalized weight Gaussian pyramid of each image, and collapse the fused Laplacian pyramid that is weighted average for each level separately by

$$
L\{F\}_{x, y}^{d}=\sum_{k=1}^{N} L\{I\}_{x, y, k}^{d} G\{W\}_{x, y, k}^{d}
$$

where $L\{I\}_{x, y, k}^{d}$ is the Laplacian pyramid of the $k$-th image on the $d$-th level, $G\{W\}_{x, y, k}^{d}$ is the normalized Gaussian pyramid of the corresponding weight map according to the weighted product of three quality measures by

$$
\begin{gathered}
W_{k, x, y}=\left[\sum_{k^{\prime}=1}^{N} \tilde{W}_{k^{\prime}, x, y}\right]^{-1} \tilde{W}_{k, x, y} \\
\tilde{W}_{k, x, y}=\left(C_{k, x, y}\right)^{w_{C}} \times\left(S_{k, x, y}\right)^{w_{S}} \times\left(E_{k, x, y}\right)^{w_{E}}
\end{gathered}
$$

where $C, S$ and $E$ are contrast, saturation, and well-exposedness, respectively with corresponding weighting exponents $w_{C}, w_{S}$ and $w_{E}$. Color images can be processed for each color channel separately.

When the dynamic range of the scene is not very large, this method works well as the input picture sequences contain enough information for fusion. However, when the dynamic range of the scene is very large and contains ultra-bright or ultra-dark areas where only a small amount of details can be captured in spite of adjusting the camera exposure parameter, the method will loss details in such areas. The ultra-light or ultra-dark area is shown in Figure 2(a). There are six input images captured in a real scene. In some areas of the scene, such as the area inside the garage, the light is so dim, then the corresponding areas in all captured images are almost darkness except that the last two images contain small amount of visual information inside the garage, we call the area ultra-dark area. Similarly, we call the area ultra-bright area when the light is strong. When blending the ultra-bright or ultra-dark area from the input images, one should give dominant weight values to the pixels of the images that contain the visual information of the area. Unfortunately, in this method the overaveraging of weight leads to loss of the dominant pixels and thus loses the visual details in ultra-bright or ultra-dark areas. The weakness of the method is exposed more and more obviously as the increase of pyramid level as shown in Figure 2.

When the pyramid level $D$ is small, the high frequency information is fused, the Gaussian kernel of the weight map pyramid only affects pixels in a small local, the weights of pixels with more information are large enough for the details preserved well. Particularly, when $D=$ 0 as shown in Figure 2(b), fusion process is equivalent to the simple weighted average, the detail information for each pixel can be preserved but the seams are obvious. With the 
increase of $D$, high-level pyramids blend the low frequency information; the Gaussian kernel involves larger areas by the recursive formula

$$
w_{d}(i, j)=\sum_{m=-2 n=-2}^{2} \sum^{2} g(m, n) w_{d-1}(2 i+m, 2 j+n)
$$

where $w_{d}$ is the weight map of the $d$-th level and $g(m, n)$ is a $5 \times 5$ Gaussian kernel window. Each weight value is more and more affected by the tremendously different adjacent pixel weights from itself, the weights of informative pixels become so smaller (the up part of Figure 2(d)) as to lose details as shown in the fourth image $(D=7)$ of Figure 2(b), the information inside the garage is almost lost compared with the last input image. It is not mentioned how to choose the appropriate pyramid level $D$ in [12]. From the illustrations of Figure 2(b), we can see that there is a contradiction on selection of $D$. The smaller value of $D$ produces more seams and halos while the larger value of $D$ leads to loss of details. Let $H$ and $W$ are the height and width of the image, respectively. In this paper, we compute $D$ by

$$
D=\left\lfloor\log _{2}[\min (H, W)]\right.
$$

to ensure no halos and introduce a novel mechanism to modify the weight map and preserve details as much as possible, which solves the contradiction above. The result of our method for this example is shown in Figure 2(c).

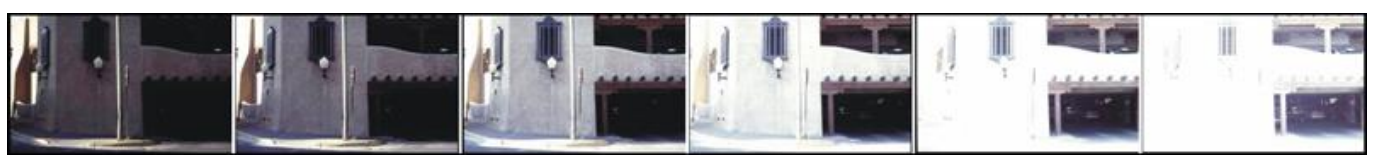

(a) Input pictures

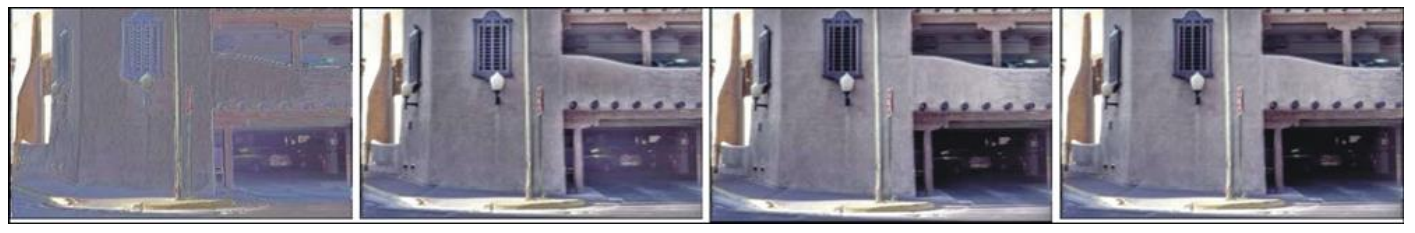

(b) Results of Mertens, et al., with different $D$

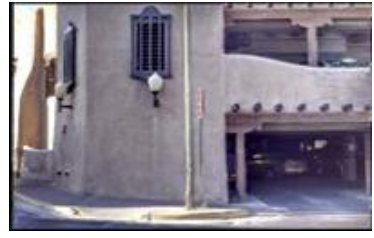

(c) Our result
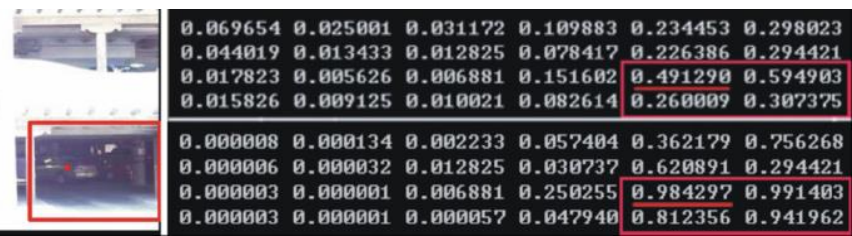

(d) Weight values comparison on the 6-th level of the sixth image

Figure 2: (b) Shows the Results of Mertens, et al., with Different Pyramid Level

$D$. From Left to Right: $D=0, D=4, D=6, D=7$, Respectively. When $D=0$, Seams are Obvious. When $D=4$, we can See the Obvious Halos Around the Iron Window. When $D=6$ or $D=7$, Halos Disappear but the Car Inside the

Garage is not Clear. In (c) we Make the Car Visible without Halos after Introducing WMF with $D=7$. (d) Shows Weight Comparison on the 6-th Level of the Sixth Image between the Weight of Mertens, et al., (up) and our Modified Weight (Bottom). The Red Point Signifies the Pixel at the Position $(64,256)$, with the Weight Modified from 0.491290 to 0.984297 (Marked with the Red Line) 


\section{Our Approach}

\subsection{Overview of our Approach}

The input exposure images are captured from a static scene with no camera motion. Our approach is a multi-resolution method, blending for each Laplacian pyramid level separately according to weight maps. The weight maps are computed guided by quality measures and modified by WMF. Finally we collapse the fused Laplacian pyramid to obtain the final image. Figure 3 shows the pipeline of our algorithm. The main difference between the method of Mertens, et al., and ours is that we propose a nonlinear power function called WMF to manipulate weight maps, preserving color and details better than previous methods without halos and distortion. We construct the Laplacian pyramid of fused image by

$$
L\{F\}_{x, y}^{d}=\sum_{k=1}^{N} L\{I\}_{x, y, k}^{d} G\left\{W^{\prime}\right\}_{x, y, k}^{d}
$$

where $G\left\{W^{\prime}\right\}_{x, y, k}^{d}$ is the normalized Gaussian pyramid of the modified weight map by

$$
\begin{gathered}
G\left\{W^{\prime}\right\}_{k, x, y}^{d}=\left[\sum_{k^{\prime}=1}^{N} G\left\{\tilde{W}^{\prime}\right\}_{k^{\prime}, x, y}^{d}\right]^{-1} G\left\{\tilde{W}^{\prime}\right\}_{k, x, y}^{d} \\
G\left\{\tilde{W}^{\prime}\right\}_{k, x, y}^{d}=G\{W\}_{k, x, y}^{d} \times G\{M\}_{k, x, y}^{d}
\end{gathered}
$$

where $G\left\{W^{\prime}\right\}_{x, y, k}^{d}$ is the WMF map Gaussian pyramid of the $k$-th image. The construction of full resolution WMF $M_{k, x, y}$ is discussed in Section 4.3.

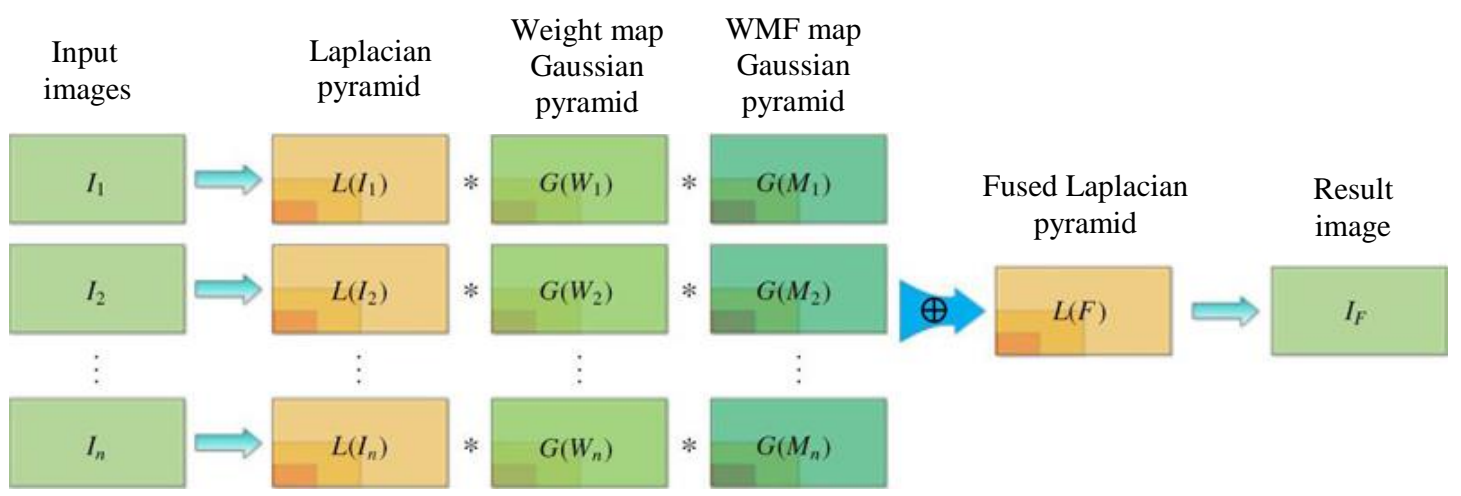

Figure 3. Pipeline of our Method

\subsection{Determination of Ultra-bright and Ultra-dark Areas}

The aim of this step is to detect the ultra-bright or ultra-dark areas where only a small amount of details exist in the input images. We find out that the average brightness of input images can reflect the luminance of the scene and meet our requirement perfectly (Figure 4, left). So we determine ultra-bright and ultra-dark areas according to the average brightness by

$$
\bar{l}_{x, y}=\frac{\sum_{k=1}^{N} l_{k, x, y}}{N}
$$

We modify the weight only for ultra-bright and ultra-dark areas and keep other normal pixels stable as much as possible. We regard pixels to be located in normal areas if $\lambda<\bar{l}_{x, y}<1-\lambda$. In this case we remain the weight of these pixels unchanged with $M_{k, x, y}=1$. Otherwise, we modify the weight map with nonlinear adjustment. The ultra-bright and ultra-dark areas can 
contain some normal pixels because this segmentation strategy is pixel-based. We deal with this situation by a constraint in Section 4.3.

Parameter $\lambda$ is variable from 0 to 0.5 . If $\lambda=0$, no ultra-bright or ultra-dark areas exist and no weight is modified. If $\lambda=0.5$, the whole scene is regarded as the ultra-bright and ultradark area. This global manipulation can weaken and counterbalance the weight modification magnitude. Based on a large number of experiments, we have $\lambda=0.2$ for all the scenes and the ultra-bright and ultra-dark areas where contain the small amount of details of the input images are well detected. (Figure 4, right)
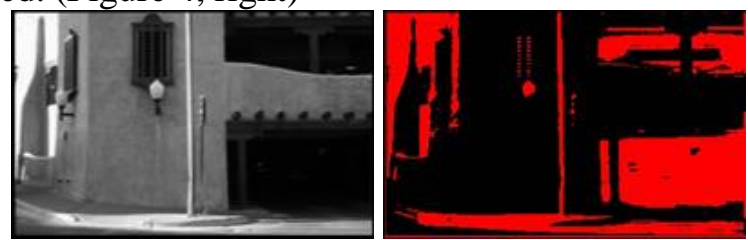

Figure 4. Average Brightness Map (left). From Dark to Bright the Brightness

Value Gradually Increases. The Segmented Areas (Right) by the Average Brightness with the Threshold Value $\lambda=0.2$, where Red Pixels Represent the Ultra-bright and Ultra-dark Areas

\subsection{Definition of WMF and Modification of Weight Maps}

We are committed to assign big weight for the pixels that contain more color and detail information in ultra-bright and ultra-dark areas. So we amplify these weight coefficients with WMF as follows:

$$
M_{k, x, y}=\left(N \cdot W_{k, x, y}\right)^{\beta}
$$

where the parameter $\beta$ controls the degree of amplification. Based on $M_{k, x, y}$, we build the Gaussian pyramids of WMF maps in the similar way except that we should always apply the constraint $G\{W\}_{x, y, k}^{d}=1$ if pixels are located in normal areas by the judgment statement $\lambda<\bar{l}_{2^{d} x, 2^{d} y}<1-\lambda$, which avoids the modified weight values being counterbalanced and disturbed at each level.

We determine the value of parameter $\beta$ by solving an objective function. We adopt the image quality metric of [18] to construct our objective function. This metric generates a distortion map by comparing the fused image with a reference image. The reference image is the HDR image from the same bracketed exposures. The distortion map is obtained by three detection probability maps, including $P_{\text {loss }}, P_{a m p l}$ and $P_{r e v}$, respectively for three types of distortions including loss of visible contrast, amplification of invisible contrast and reversal of visible contrast. We equally treat the three distortion detection probability by computing their average value as $P_{x, y}(\beta)$ to represent magnitude of distortion probability at $(x, y)$ with the parameter $\beta$. We compute the average probability for all pixels to measure the distortion by

$$
\bar{P}(\beta)=\frac{\sum_{x=1}^{H} \sum_{y=1}^{W}\left[P_{x, y}(\beta)\right]}{\sum_{x=1}^{H} \sum_{y=1}^{W}}
$$

Figure 5(a) shows the function curves of $\bar{P}(\beta)$ for Figure 2 and Figure 6. We can see that when $\beta=0$, the magnitude of distortion probability is largest. With the increase of $\beta$, the magnitude of probability $\bar{P}(\beta)$ is decreased. It means that the distortion between the test image and the reference image is decreased gradually. However, it tends to approximate to constant when $\beta$ increases to a certain value $\beta_{m}$. We actually aim to search $\beta_{m}$ to ensure the 
lowest distortion. In order to compute $\beta_{m}$ precisely, we compute the absolute value of first derivative of $\bar{P}(\beta)\left(\left|\bar{P}^{\prime}(\beta)\right|\right)$ as our objective function and find out the minimum $\beta$ as $\beta_{m}$ to meet the condition $\left|\bar{P}^{\prime}(\beta)\right|<\varepsilon$, where $\varepsilon$ is a very small value near 0 and we set $\varepsilon=0.001$. We get $\beta_{m}=8.5$ for Figure 2 and $\beta_{m}=5.0$ for Figure 6 by iterative computation method. Their function curves of $\left|\bar{P}^{\prime}(\beta)\right|$ are shown in Figure 5(b).
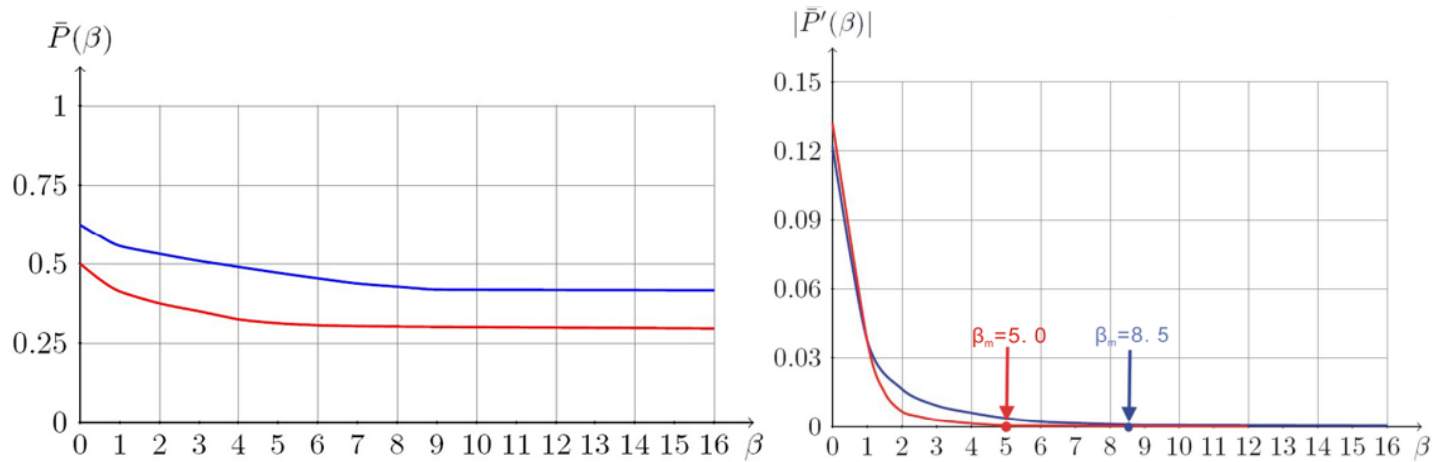

Figure 5. Function Curves of $\bar{P}(\beta)$ and $\left|\bar{P}^{\prime}(\beta)\right|$ for Figure 2 (blue, $\boldsymbol{\beta}_{\boldsymbol{m}}=8.5$ ) and Figure $6\left(\mathrm{red}, \beta_{m}=5.0\right)$

Based on the above parameter calculation for a large number of scenes, we find that for most scenes $\beta_{m}$ is less than 10 , such as $\beta_{m}=7.2$ for Figure $1, \beta_{m}=3.5$ for Figure $8, \beta_{m}=6.3$ for Figure 9 and so on. Figure 7 which shows different results under variations of $\beta$. Therefore, we set the parameter $\beta$ large enough for $\beta>\beta_{m}$ with no need to calculate $\beta_{m}$ for every scene. ( $\beta=15$ is suitable for most scenes in this paper)

With the weight manipulation by WMF, the pixels that contain more details information have an absolute advantage over other pixels. Even if under the weight smooth effect of Gaussian pyramid level by level, at the high-level pyramid these pixels can still keep the weight advantage. We pick out a single representative pixel weight value for better description of the problem. We choose the pixel at the position $(64,256)$ in the garage image sequence (Figure 2(d)). Because the detail information of this pixel is mainly embodied in the sixth garage image, we desire to keep the weight advantage over other five images at each pyramid level. When $D=0$, the weights of 6 input images for this pixel are 0.000000 , $0.000000,0.000000,0.000215,0.065051,0.934734$, where the sixth weight 0.934734 has reached the advantage large enough for details preserved. However, the weight advantage reduces gradually with the increase of $D$ for the weight smooth of Gaussian kernel. We can compute the corresponding sixth weight when $D=6$ at the position $(1,4)$, given by $\left(64 / 2^{6}\right.$, $256 / 2^{6}$ ), equal to 0.491290 by Eq. 4 . The weight advantage is not large enough and causes details lost. After the weight modification by our method, the corresponding normalized sixth weight when $D=6$ at $(1,4)$ is increased to 0.984297 which achieves large enough weight advantage. Similarly, the four weight values in the red box of Figure 2(d) are greatly improved.

\section{Experiment Results and Comparisons}

We compute the pyramids based on a $5 \times 5 \mathrm{kernel}$, on a $2.33 \mathrm{GHz}$ Intel core 2 Duo CPU, 1.96 GB memory. In the scene with very large dynamic range or the exposure adjustment range is narrow, only a small amount of details are captured in the ultra- 
bright and ultra-dark areas. Our method could preserve the details as much as possible in these areas while Mertens, et al., will lose these details, such as the ultra-dark garage (Figure 2(c)), and the details in the ultra-bright hall (Figure 9). Especially, for a large number of the multi-exposure input images, our method has larger advantage over [12] on the effect of details capture, for example, fusing 20 input exposures in Figure 10. In other words, our result image fused with fewer input pictures contains amount of detail information comparable to Mertens, et al., with more input pictures (Figure 6). We prove the effectiveness of our method by several quality assessment metrics.

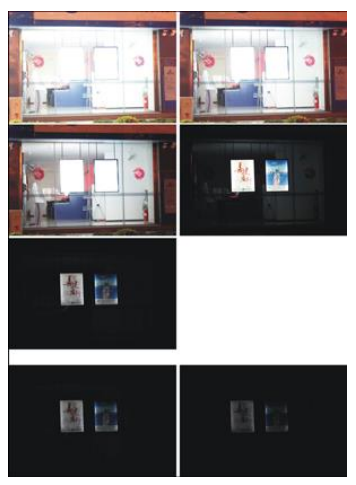

(a)

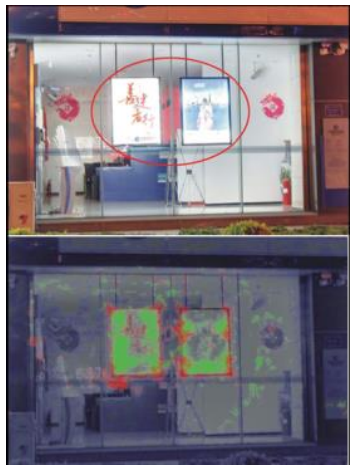

(b)

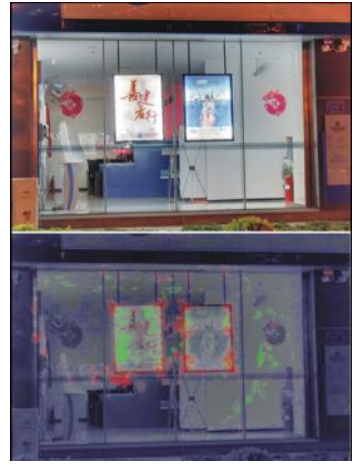

(c)

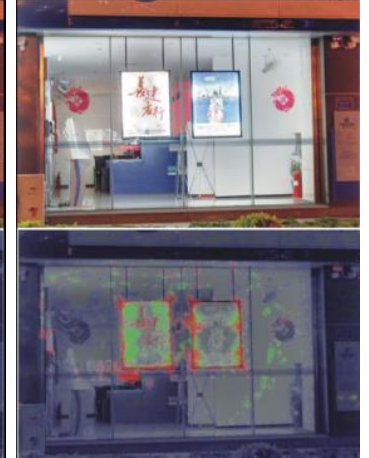

(d)

Figure 6. (a) Five Exposures and Two Additional Exposures (Bottom). (b) and (d) are Results of Mertens, et al., by Five Exposures and by all Seven Exposures. (c) Is our Result by Five Exposures and Contains more Details than (b) and (d). The Distortion Map of (c) Contains Less Green Color on the Two Billboards than (b) and (d)

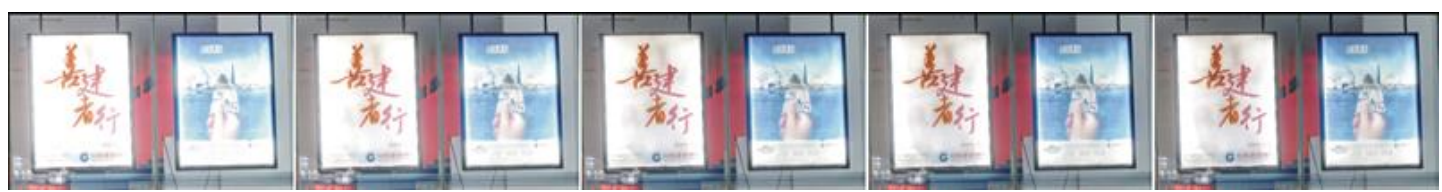

Figure 7. Close-up of Figure 6 which shows Different Results Under

Variations of $\beta$ From Left to Right: $\beta=1.5(\bar{P}(\beta)=0.383), \beta=3.5(\bar{P}(\beta)=0.329)$,

$$
\beta=5.0(\bar{P}(\beta)=0.306), \beta=10(\bar{P}(\beta)=0.302) \text { and } \beta=25(\bar{P}(\beta)=0.301) \text {, }
$$

Respectively. The Distortion Decreases Dramatically when $\beta<\beta_{m}\left(\beta_{m}=5.0\right)$ but with Little Variation when $\beta>\beta_{m}$.

\subsection{Assessment by Distortion Maps}

We visualize the three types of distortion with three RGB color channels [18]. The saturation of each color indicates the magnitude of distortion probability. In all the distortion maps of this paper, we chose green for loss of visible contrast, blue for amplification of invisible contrast, and red for reversal of visible contrast. The parameters to generate the distortion map are as follows: All LDR images are shown in a typical display with maximum luminance 100 and gamma 2.2, the pixels per visual degree is 30 and the observer distance is 0.5 meters. Our distortion map contains dramatically less green color than Mertens, et al., [12] in ultra-bright and ultra-dark areas without introducing more blue and red color (see Figure 6 and Figure 9). Figure 11 shows the comparison with several other exposure fusion 
methods. The corresponding distortion maps manifest that our result causes less visible detail lost.

\subsection{Comparisons under Different Measure Exponents}

We use equally weighted quality measures with $w_{C}=w_{S}=w_{E}=1$ in Eq. 3 for all scenes of this paper unless noted otherwise. Adjusting the three quality measure exponents can lead to slight variation, but under the same measure exponents our method can preserve more details than Mertens et al. (Figure 8).

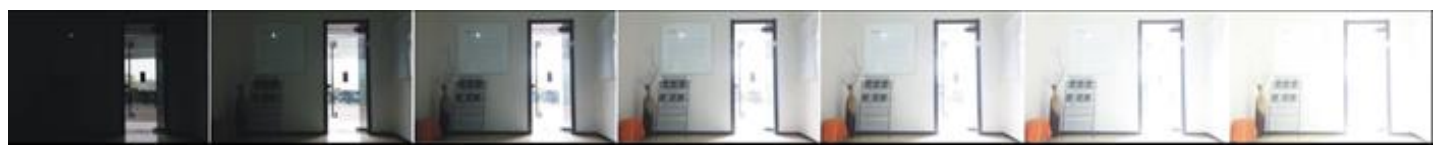

(a) Seven input pictures

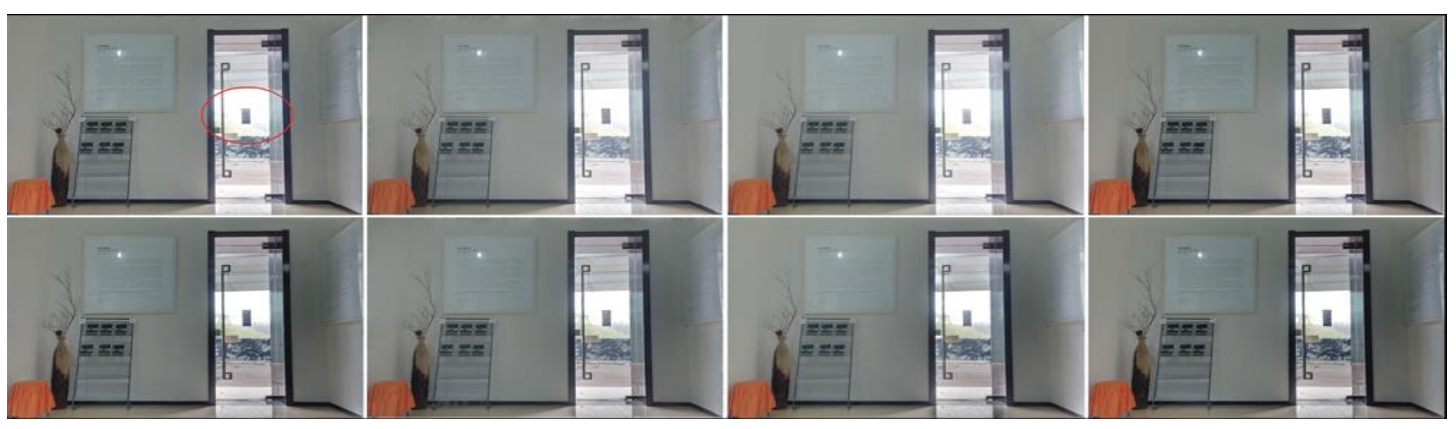

(b) Comparisons of Mertens, et al., and our result with different weights setting

Figure 8. (b) Shows Comparisons of Mertens, et al., (the Top Row) and our Result (the Bottom Row) by Setting Different Quality Measure Weights. From Left to Right: $w_{C}=w_{S}=w_{E}=1$ for the First Column, $w_{C}=1 ; w_{S}=w_{E}=0$ for the Second Column, $w_{S}=1 ; w_{C}=w_{E}=0$ for the Third Column, $w_{E}=1 ; w_{C}=w_{S}=0$ for the Fourth Column, Respectively. The Faraway Trees outside the Art Gallery Door in our Result are Clearer for Each Column

\subsection{Assessment by Average Quality Measures}

We also make quality assessment according to the average objective quality measure by

$$
Q A=\frac{1}{H \cdot W} \sum_{x=1}^{H} \sum_{y=1}^{W} Q_{x, y}
$$

where $Q_{x, y}$ is one of the three quality measures including contrast, saturation and wellexposedness defined in [12]. The value of quality assessment $Q A$ including average contrast, average saturation and average well-exposedness, have been improved as shown in Table 1, which further prove the high quality of our method by quantitative measurement.

Table 1. Objective Quality Assessment QA Comparisons of Different Scenes between Mertens, et al., (M1) and our Result (M2)

\begin{tabular}{|c|c|c|c|c|c|c|}
\hline Quality measure & \multicolumn{2}{|c|}{ Contrast } & \multicolumn{2}{c|}{ Saturation } & \multicolumn{2}{c|}{ Well-exposedness } \\
\hline & M1 & M2 & M1 & M2 & M1 & M2 \\
\hline Figure 2 & 18.098 & 19.286 & 7.182 & 7.632 & 0.493 & 0.508 \\
\hline Figure 11 & 17.986 & 19.261 & 23.589 & 25.034 & 0.178 & 0.210 \\
\hline Figure 6 & 6.060 & 6.274 & 15.351 & 15.700 & 0.274 & 0.340 \\
\hline
\end{tabular}




\section{Conclusion}

In this paper, we obtain a detail-preserving and visually natural image in a multiresolution fashion by fusing a sequence of multi-exposure images directly. We propose WMF to modify weight maps, increasing the weight of informative pixels in ultrabright and ultra-dark areas and preserving detail information. Our approach has a wider adaptability to scenes with very large dynamic range. The quality of our method is superior to previous exposure fusion methods and existing tone mapping methods.

We would like to deal with the fusion process with GPU implementation to further improve the efficiency for real-time display. We also would like to explore more effective weight modification strategies. Finally, we would like to make our work more adaptable to the scenes with noise and dynamic scenes with objects or camera motion.

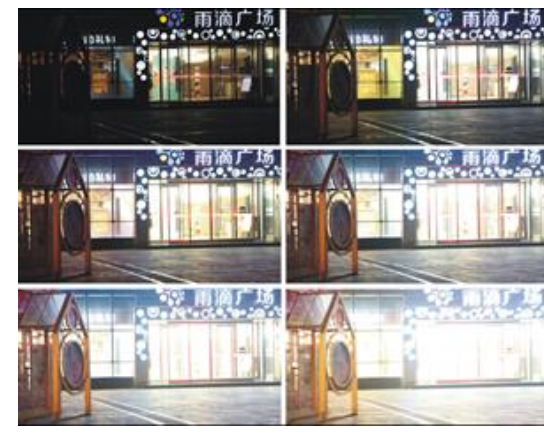

(a) Six input pictures

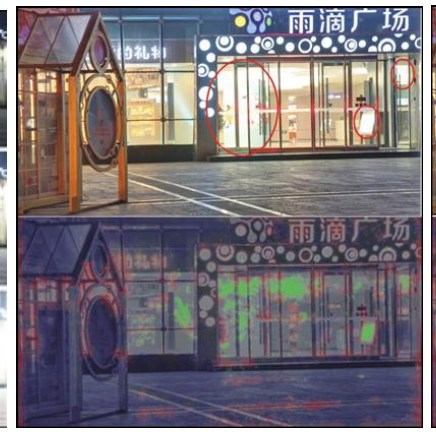

(b) Mertens et al.

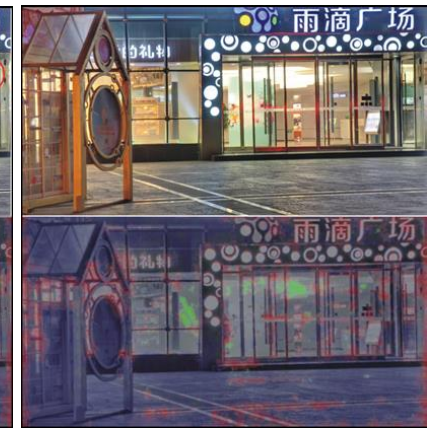

(c) Ours

Figure 9. Our Result (c) Looks much Clearer Inside the Hall than (b). The Corresponding Distortion Maps show our Result Causes less Detail Lost (Green)

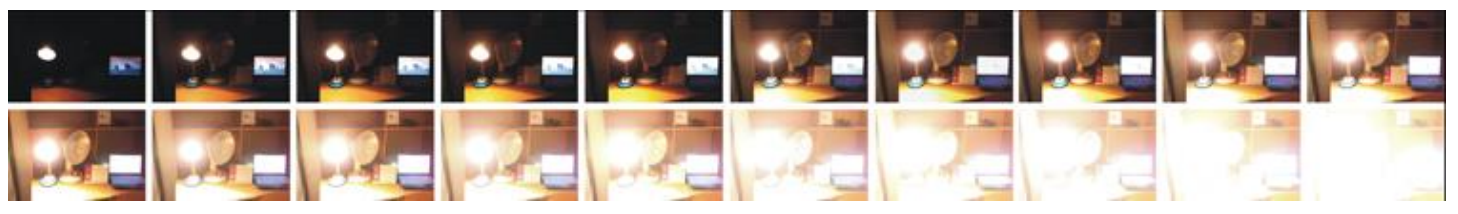

(a) Twenty input pictures

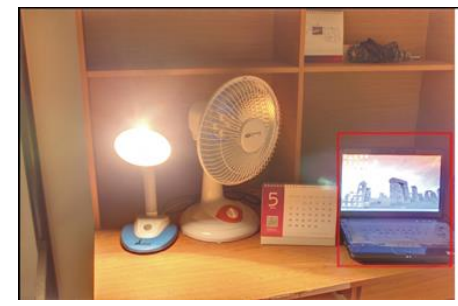

(b) Mertens, et al.,

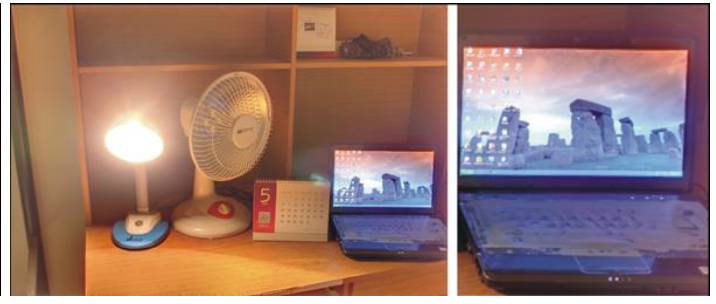

(c) Ours

Figure 10. Only the First Several Input Pictures Contain useful Details on the Laptop Screen. Our Result (c) Preserves these Details as Many as Possible but (b) Loses them 


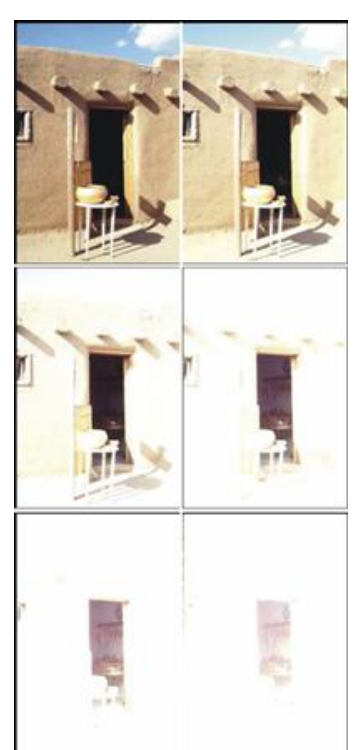

Input pictures

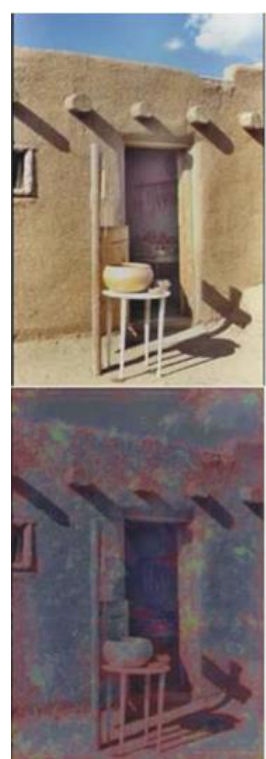

Goshtasby

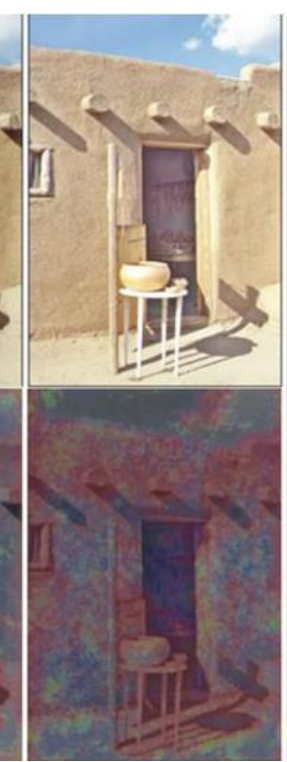

Wei Zhang, et al.

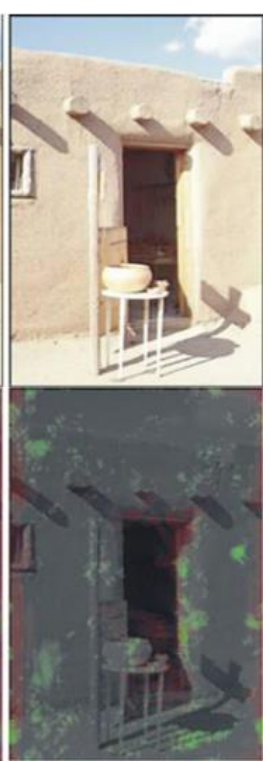

Raman, et al.,

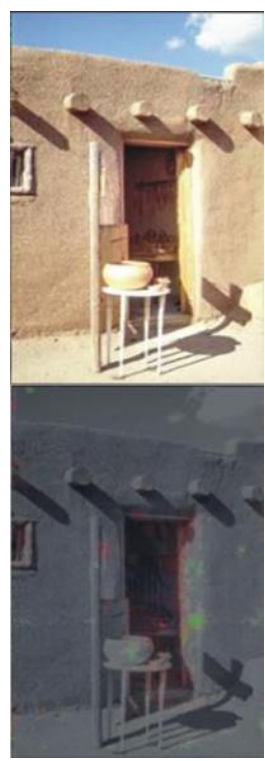

Ours

Figure 11. Comparisons of other Main Fusion Methods (top) with the Corresponding Distortion Maps (Bottom). The Results from Left to Right: Result of Goshtasby [11], Wei Zhang, et al., [15], Raman, et al., [13] and ours, Respectively Our Result causes Less Visible Detail Lost (Green) on the Cave Wall and not so much Contrast Amplification (Blue) and Reversal (Red) as $[11,15]$

\section{Acknowledgements}

This research was supported by NSFC-Guangdong Joint Fund Project (U1035004) and Graduate Innovation Foundation of Shandong University (GIFSDU) yzc12140.

\section{References}

[1] R. Erik, W. Greg, P. Sumanta, D. Paul, H. Wolfgang and M. Karol, "High Dynamic Range Imaging”, Second Edition: Acquisition, Display, and Image-Based Lighting, (2010).

[2] B. Francesco, A. Alessandro, D. Kurt and C. Alan, "Advanced High Dynamic Range Imaging", Theory and Practice, (2011).

[3] A. Manoj and N. Ahuja, "Split Aperture Imaging for High Dynamic Range", International Journal of Computer Vision, vol. 58, no. 7, (2004).

[4] H. Seetzen, W. Heidrich and W. Stuerzlinger, "High dynamic range display systems", ACM Transactions on Graphics, vol. 23, no. 760, (2004).

[5] M. D. Tocci, K. Chris, T. Nora and S. Pradeep, “A versatile HDR video production system”, ACM Trans. Graph., vol. 30, no. 1, (2011).

[6] P. E. Debevec and M. Jitendra, "Recovering high dynamic range radiance maps from photographs", ACM SIGGRAPH, (1997).

[7] F. Durand and J. Dorsey, "Fast bilateral filtering for the display of high-dynamic-range images", ACM SIGGRAPH, (2002).

[8] R. Erik, S. Michael, S. Peter and F. James, "Photographic tone reproduction for digital images", ACM SIGGRAPH, (2002).

[9] F. Raanan, L. Danin and W. Michael, "Gradient domain high dynamic range compression", ACM SIGGRAPH, (2002).

[10] F. Drago, K. Myszkowski, T. Annen and N. Chiba, “Adaptive Logarithmic Mapping For Displaying High Contrast Scenes", Computer Graphics Forum, vol. 22, no. 419, (2003).

[11] A. Goshtasby, "Fusion of multi-exposure images", Image and Vision Computing, vol. 23, no. 611, (2005). 
[12] T. Mertens, J. Kautz and F. V. Reeth, "Exposure Fusion: A Simple and Practical Alternative to High Dynamic Range Photography”, Computer Graphics Forum, vol. 28, no. 161, (2009).

[13] S. Raman and S. Chaudhuri, "Bilateral Filter Based Compositing for Variable Exposure Photography", In Short Papers, Euro graphics, (2009).

[14] Z. Ying, S. Jianbing and H. Ying, "Subband Architecture Based Exposure Fusiony", 2010 Fourth PacificRim Symposium on Image and Video Technology, (2010).

[15] W. Zhang and W.-K. Cham, "Gradient-directed composition of multi exposure images", Computer Vision and Pattern Recognition, (2010).

[16] R. Erik and D. Kate, "Dynamic Range Reduction Inspired by Photoreceptor Physiology", IEEE Transactions on Visualization and Computer Graphics, (2005).

[17] P. Burt and E. Adelson, "The Laplacian Pyramid as a Compact Image Code", IEEE Transactions on Communications, (1983).

[18] T. O. Aydin, R. Mantiuk, K. Myszkowski and H.-P. Seidel, "Dynamic range independent image quality assessment", ACM SIGGRAPH, (2008).

\section{Authors}

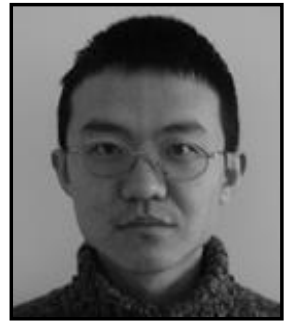

Chunmeng Wang, he received the B.S. degree in Department of Software Engineering from Shandong University, Jinan, China, in 2009, and he is currently a Ph.D. student in the lab of Humancomputer interaction and virtual reality in the department of Computer Science and Technology at Shandong University. His research interests include computer graphics, image processing, high dynamic range image.

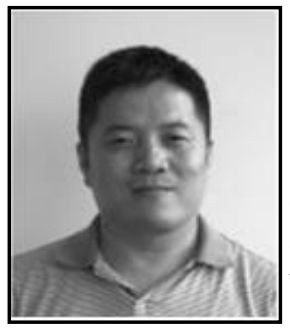

Changhe Tu, he is a Professor of School of Computer Science and Technology at Shandong University. He obtained his $\mathrm{PhD}$ in 2003 from Shandong University. His research interests include geometric modeling and processing, computer aided geometric design, computer graphics and computer animation. His publications cover such topics as analysis and computation of intersections between two quadric surfaces, distance computation of curved surfaces, mesh processing, and facial expression tracking. He is a member of CCF (China Computer Federation) and ACM. 